\title{
e-Boletim de Física
}

International Centre for Condensed Matter Physics

Instituto de Física, Universidade de Brasília

Ano V, Fevereiro de $2016 \bullet$ http://periodicos.unb.br/index.php/e-bfis • eBFIS 5 1401-1(2016)

\section{Nonlinear dynamical systems seen through the scope of the Quasi-polynomial theory}

\author{
Léon Brenig* \\ Brussels University (ULB). Faculty of Sciences. Theoretical and Mathematical Physics Department. 1050 Brussels, Belgium.
}

\begin{abstract}
A unified theory of nonlinear dynamical systems is presented. The unification relies on the Quasipolynomial approach of these systems. The main result of this approach is that most nonlinear dynamical systems can be exactly transformed to a unique format, the Lotka-Volterra system. An abstract Lie algebraic structure underlying most nonlinear dynamical systems is found. This structure, based on two sets of operators obeying specific commutation rules and on a Hamiltonian expressed in terms of these operators, bears a strong similarity with the fundamental algebra of quantum physics.

From these properties, two forms of the exact general solution can be constructed for all LotkaVolterra systems. One of them corresponds to a Taylor series in power of time. In contrast with other Taylor series solutions methods for nonlinear dynamical systems, our approach provides the exact analytic form of the general coefficient of that series. The second form of the solution is given in terms of a path integral. These solutions can be transformed back to solutions of the general nonlinear dynamical systems.
\end{abstract}

Keywords: Nonlinear systems.

\section{INTRODUCTION}

This series of two lectures was dedicated to the Quasipolynomial (QP) approach to the theory of Nonlinear Dynamical Systems, i.e. systems of nonlinear ordinary differential equations that frequently appear in mathematical models in Physics, Chemistry, Biology and other scientific disciplines.

Though a great diversity of dynamical systems are used in scientific modelling, most of them can be cast in the form of systems of ordinary differential equations with polynomial or, more generally, quasi-polynomial nonlinearities. The latter are linear superposition of monomials with exponents that can be non-integers, real numbers. These monomials represent the interactions between the entities that are represented by the dynamical variables.

The Quasi-Polynomial approach relies on a special notation for writing the quasi-polynomials that appear in the vector field of a given dynamical system. Using that notation it is possible to show that any quasi-polynomial dynamical system can be exactly transformed to a LotkaVolterra (LV) system of differential equations. This property reduces the task of solving, completely or partially, nonlinear dynamical systems to the task of solving the corresponding LV systems. Solving a LV system amounts to solve the infinite set of QP systems that are equivalent to that LV system.

\footnotetext{
* lbrenig@ulb.ac.be
}

We, thus, focus on the LV systems. Being a particular form of quadratic systems, they represent in fact the lowest degree of nonlinearity. We find an abstract Lie algebraic structure underlying these systems. A Hamiltonian is shown to generate the time evolution of any given LV system. We give three explicit realizations of the LV algebra. One of them is implemented with boson creation-destruction operators. In this framework, the Hamiltonian is expressed in terms of creation-destruction operators but is not Hermitian. Another realization leads to the Liouville partial derivative equation that represents the conservation of the probability density norm. This equation describes the time evolution of the probability density when the initial condition of the LV system is only known in probability. The abstract Lie algebraic of LV systems is then compared with that of linear systems. It can be seen that the difference between the two types of systems lies essentially in the different Hamiltonians that generates them.

Finally, we show that two forms of the exact general solution can be constructed for LV systems. They are based on two different realizations of the abstract LV algebra. One form of the solution is given in terms of a Taylor series in power of time. In contrast with other Taylor series solutions methods used for nonlinear dynamical systems, here we find the exact analytic form of the general coefficient of that series. The second form of the solution is given in terms of a path integral quite similarly with quantum field theory.

Finally, there remains an important open question: What happens to these solutions when the dynamical 
system is in a chaotic regime?

This work results from a long-standing collaboration with Drs. Tarcisio Marciano da Rocha Filho (UnB, Brasilia), Annibal Figueiredo (UnB, Brasilia) and Iram Gléria (Federal University of Alagoas), and with Drs. Victor Fairén (UNED, Madrid), Benito HernándezBermejo (URJC, Madrid) and Rubén Diaz-Sierra (UNED, Madrid). The first and crucial steps have been made with Dr. Alain Goriely (Oxford University) who was, at that epoch, doing his $\mathrm{PhD}$ with me. Many thanks to all of them for our collaboration and friendship.

Outline

\section{Lecture 1:}

- Linear versus nonlinear Dynamical Systems (DS)

- Examples

- Quasi-Polynomial (QP) notation of DS

- Examples of DS in QP format

- Quasi-monomial transformations

- Transformation of QP systems into Lotka-Volterra (LV) canonical form

- Extension of the set of DS reducible to QP systems

- Applications: Invariants, dimensional reduction, stability

\section{Lecture 2:}

- Carleman infinite embedding representation of LV dynamics

- Abstract Lie algebraic structures of LV systems

- Two other realizations of the abstract algebra: creation-destruction operators and Liouville

- Algebraic difference between linear and nonlinear DS

- General solution via Taylor series

- General solution via path integrals

- Conclusions

\section{LECTURE 1}

\section{A. Linear versus nonlinear dynamical systems}

Dynamical systems are everywhere. They appear in:

Classical mechanics: Newton or Hamilton equations Fluid dynamics: Fourier modes of Navier-Stokes eqs Plasma physics and nonlinear optics: Coupled waves eqs
Electronics: Kirchhoff equations of circuits Chemistry: Rate equations for chemical reactions Biology-Ecology: Populations dynamics equations Economy: rate equations for economic variables

Their general form is:

$$
\begin{aligned}
& \frac{d X_{1}}{d t}=f_{1}\left(X_{1}(t), \ldots, X_{n}(t)\right) \\
& \frac{d X_{2}}{d t}=f_{2}\left(X_{1}(t), \ldots, X_{n}(t)\right) \\
& \vdots \\
& \frac{d X_{n}}{d t}=f_{n}\left(X_{1}(t), \ldots, X_{n}(t)\right),
\end{aligned}
$$

where the variable $t$ is time and where the functions $f_{i}\left(X_{1}, \ldots, X_{n}\right)$ represent the interactions between the variables . Let us compare the main features of linear and nonlinear dynamical systems (DS):

\section{Linear DS:}

$$
\dot{x}_{i}=\sum_{j=1}^{n} L_{i j} x_{j} \quad i=1, \ldots, n,
$$

with $\dot{x}_{i}=d x_{i} / d t$ and where $L$ is a constant real $n x n$ matrix.

The system of equations (1) is form-invariant under linear transformation:

$$
x_{i}^{\prime}=\sum_{j=1}^{n} T_{i j} x_{j} \quad i=1, \ldots, n .
$$

Indeed, transformation (2) acting on equation (1) leads to:

$$
\dot{x}_{i}^{\prime}=\sum_{j=1}^{n} L_{i j}^{\prime} x_{j}^{\prime} \quad i=1, \ldots, n
$$

with:

$$
L^{\prime}=T \circ L \circ T^{-1},
$$

where $\circ$ denotes the matrix product. Form-invariance means that equation (1) and its transformed version (3) have the same linear shape. The only difference is that the two matrices L and L' are different but they are related though relation (4).

The interest of this property is that one can find a particular matrix $\mathrm{T}$ that put $\mathrm{L}$ ' into diagonal or Jordan form. From this one can easily get the general solution to equation (1). This is the origin of the efficiency of linear algebra in solving linear dynamical systems like (1).

\section{Nonlinear DS:}

$$
\dot{x}_{i}=f_{i}\left(x_{1}(t), \ldots, x_{n}(t)\right) \quad i=1, \ldots, n .
$$

In contrast to the above linear DS, no general analytic method for solving equations (5) was until now available. This is due to infinite diversity of functional dependence that can have the nonlinear functions . As 
a consequence, no form-invariance property similar to the above for linear DS was known before the advent of the quasi-polynomial theory presented in the sequel of this article. The unavailability of a general solution for most nonlinear DS led to the development of qualitative methods and approximation techniques such as linear stability analysis, bifurcation theory, Lyapunov exponents and the theory of chaotic attractors, numerical integration.

One should also keep in mind that most linear DS in scientific models are approximations of nonlinear DS.

\section{B. Examples of nonlinear DS:}

The asymmetric Euler top and also the three waves model in plasma physics, hydrodynamics and nonlinear optics:

$$
\begin{aligned}
& \dot{x}_{1}=-a_{1} x_{1}+\mu x_{2} x_{3} \\
& \dot{x}_{2}=-a_{2} x_{2}+\beta x_{1} x_{3} \\
& \dot{x}_{3}=-a_{3} x_{3}+\gamma x_{1} x_{2} .
\end{aligned}
$$

The Lorentz model was derived from the Fourier modes transformation of the hydrodynamic equations of thermal convection:

$$
\begin{aligned}
& \dot{x}_{1}=\sigma\left(x_{2}-x_{1}\right) \\
& \dot{x}_{2}=\rho x_{2}-x_{2}-x_{1} x_{3} \\
& \dot{x}_{3}=x_{1} x_{2}-\beta x_{3} .
\end{aligned}
$$

The above models have nonlinearities that are at most quadratic but many other models, for example some chemical reaction rate equations, have nonlinearities with higher degree monomials or even non-integer degree monomials, i.e., quasi-monomials.

\section{Quasi-Polynomial (QP) notation of dynamical systems}

Any dynamical system with polynomial nonlinearities of integer or non-integer degrees can be written in the following manner [1]:

$$
\dot{x}_{i}=\alpha_{i} x_{i} \sum_{j=1}^{N} A_{i j} \prod_{k=1}^{n} x_{k}^{B_{j k}} \quad i=1, \ldots, n,
$$

where:

- the coefficients $A_{i j}$ are real constant entries of a $n \times N$ rectangular matrix $A$

- the exponents $B_{i j}$ are real constant entries of a $N \times$ $n$ rectangular matrix $B$

- the $\mathrm{i}$ are real constant components of a $\mathrm{n}$ dimensional vector
- $\mathrm{n}$ is the dimension of the DS (8)

- $\mathrm{N}$ is the total number of different (quasi-) monomials of form $\prod_{k=1}^{n} x_{k}^{B_{j k}}$.

It should be stressed that any polynomial or quasipolynomial function can be written in the form of the right-hand-side of equation (8). This is due to the fact that the pre-factor $x_{i}$ in the right-hand-side of (8) can be absorbed by a factor $x_{i}^{-1}$ that can appear in some monomials $\prod_{k=1}^{n} x_{k}^{B_{j k}}$ depending on the particular form of matrix $B$.

In fact, equation (8) is a way to introduce a novel notation, the so-called quasi-polynomial notation, that permits to define the above matrix $\mathrm{A}$ of coefficients and the matrix B of exponents appearing in equation (8). As we show below, these matrices play a fundamental role in the quasi-polynomial theory of nonlinear DS.

\section{Examples of dynamical systems written in the QP notation}

- The Euler asymmetric top (or three-waves model) (6) given above can be written in the QP notation (8) with:

$A=\left(\begin{array}{ccc}\mu & 0 & 0 \\ 0 & \beta & 0 \\ 0 & 0 & \gamma\end{array}\right) \quad B=\left(\begin{array}{ccc}-1 & 1 & 1 \\ 1 & -1 & 1 \\ 1 & 1 & -1\end{array}\right) \quad \alpha=\left(\begin{array}{l}-a_{1} \\ -a_{2} \\ -a_{3}\end{array}\right)$

This is an example where, due to the particular form of matrix, the pre-factor $x_{i}$ disappears due to cancellation with the inverses of these monomials. Here $\mathrm{A}$ and $\mathrm{B}$ are square matrices.

- The Lorenz system (7) takes the QP form (8) with:

$A=\left(\begin{array}{cccc}1 & 0 & 0 & 0 \\ 0 & \rho & -1 & 0 \\ 0 & 0 & 0 & 1\end{array}\right) \quad B=\left(\begin{array}{ccc}-1 & 1 & 0 \\ 1 & -1 & 0 \\ 1 & -1 & 1 \\ 1 & 1 & -1\end{array}\right) \quad \alpha=\left(\begin{array}{c}-\sigma \\ -1 \\ -\beta\end{array}\right)$

Obviously, here A and B are rectangular matrices.

\section{E. Quasi-monomial transformations}

The quasi-monomial transformations are defined by:

$$
\dot{X}_{i}=\prod_{k=1}^{n} X_{k}^{\prime C_{j k}} \quad i=1, \ldots, n,
$$

where the real constant exponents $C_{i k}$ constitute a square $n x n$ matrix $C$. This matrix must be invertible. To each matrix $\mathrm{C}$ of $\mathrm{GLn}(\mathrm{R})$ corresponds one such transformation. The set of all these transformations forms a group under the composition product that is isomorphic to $\mathrm{GLn}(\mathrm{R})$. More precisely, to avoid singularities, we restrict our scope to equations (8) for which the variables $x_{i}(t)$ remain in the positive orthant. 
Clearly, transformation (11) is a linear relation, expressed with matrix $\mathrm{C}$, between the logarithms of the new and old variables $x_{i}$ and $x_{i}^{\prime}$. This particular role of the logarithm of the variables is related to the pre-factor $x_{i}$ appearing in the QP format (8). Dividing the two sides of equation (8) by the pre-factor permits, indeed, to re-write (8) with the logarithmic time derivative of $x_{i}$. This fact plays a fundamental role in the QP theory.

Transformation (11) brings equation (8) into:

$$
\dot{x}_{i}^{\prime}=\alpha_{i}^{\prime} x_{i}^{\prime} \sum_{j=1}^{N} A_{i j}^{\prime} \prod_{k=1}^{n} x_{k}^{\prime} B_{j k}^{\prime}
$$

with :

$$
\begin{aligned}
\alpha^{\prime} & =C^{-1} \circ \alpha \\
A^{\prime} & =C^{-1} \circ A \\
B^{\prime} & =B \circ C,
\end{aligned}
$$

where $\circ$ is the matrix product.

A comparison between equations (8) and (12) shows the form-invariance of the former under transformations (11). This bears some analogy with the form-invariance of the linear DS under linear transformations we mentioned above. However, in contrast with the latter, this does not lead to any general diagonalization or Jordan form for system (12). Transformations (13) are, consequently, less powerful than the linear transformations for linear DS.

However, the transformations (11) and (13), lead to a new and important result. By choosing a particular matrix $\mathrm{C}$ for the transformation (11), the QP system (12) is shown to reduce to a Lotka-Volterra system as we show in the next chapter. This, in turn, brings a wealth of new results on the original system (8).

\section{F. Transformation of QP systems into Lotka-Volterra (LV) canonical form}

Let us start with the demonstration for the particular case of QP systems (8) with $n=N$, i.e., systems where the number $\mathrm{N}$ of independent monomials is equal to the dimension $n$ of the system. Such systems are, of course, by no means general but the proof of their transformation to the LV form is quite easy. The general case with is similar but needs a preliminary embedding or projection as we shall discuss after proving the case .

So, let us first consider the system with $N=n$ :

$$
\dot{x}_{i}=\alpha_{i} x_{i} \sum_{j=1}^{n} A_{i j} \prod_{k=1}^{n} x_{k}^{B_{j k}} \quad i=1, \ldots, n .
$$

In this case the matrix $\mathrm{B}$ of exponents is a square matrix ( $\mathrm{A}$ is also square). Let us, furthermore, assume that $\mathrm{B}$ is invertible. We, then, perform on (14) a particular quasimonomial transformation (11) with $C=B^{-1}$. Inserting this form of $\mathrm{C}$ in equations (13) we see that:

$$
\begin{aligned}
& \alpha^{\prime}=B \circ \alpha=\lambda \\
& A^{\prime}=B \circ A=M \\
& B^{\prime}=B \circ B=I .
\end{aligned}
$$

The transformed system (12), thus, becomes in this case:

$$
\dot{x}_{i}^{\prime}=\lambda x_{i}^{\prime}+x_{i}^{\prime} \sum_{j=1}^{N} M_{i j} x_{j}^{\prime} \quad i=1, \ldots, n .
$$

Due to the last equation of (15), $B^{\prime}=I$, the monomials in the transformed form of (14) are reduced to just the variables $x_{i}^{\prime}$ themselves. Thus, the transformed version of (14) becomes the equation (16) that contains only particularly simple quadratic nonlinearities.

Expression (16) is precisely the form of the so-called Lotka-Volterra dynamical systems 2, 3. The constant coefficient $M_{i j}$ describes the interaction between the variables $x_{i}^{\prime}$ and $x_{j}^{\prime}$. The $M_{i j}$ constitute the entries of a $\mathrm{n} \mathrm{x}$ n square matrix $\mathrm{M}$ that is given by $M=B \circ A$, i.e., the matrix product of the exponents matrix and the coefficients matrix of the original QP system (14). Similarly, the new coefficients of the linear term in (16) are the components of the vector $\lambda=B \circ \alpha$ where $\alpha$ is the vector of coefficients of the linear term in the original system (14).

A remarkable feature is that the quite general nonlinearity of equation (14) is reduced to the simplest form of quadratic nonlinearity in (16). A wealth of mathematical results on the LV equations exists in the literature (see for example 2, 3]). These results can be transferred to all the QP systems that are equivalent to a given LV system by performing the inverse of the transformation (11). Among them are many stability properties that can be deduced from the existence of a Lyapunov function for the LV systems.

Furthermore, all the systems having matrices A, B and vector $\alpha$ such that their product $B \circ A$ and $B \circ \alpha$ are identical, transform into the same LV system. We can, thus, say that they are all equivalent. They constitute an equivalence class. Since the transformation (11) with $C=B^{-1}$ is a diffeomorphism, we know that the trajectories of all these systems in phase-space are transformed by a diffeomorphism into the trajectories of the corresponding LV system. Hence, we can state the proposition that the set of all QP dynamical systems is divided into equivalence classes that are, each, characterized by one canonical member, the LV system. Let us stress that since most nonlinear dynamical systems can be brought to the QP form [4, this proposition is quite powerful.

For the more general systems (8) with $n \neq N$ we refer for more details to the article 5 where the demonstration of the transformation to the LV format has been given for all the three cases. However, as a summary we can say the following. For systems with $N>n$, one must first add $N-n$ new variables to the system (8). These variables obey:

$$
\frac{d X_{r}(t)}{d t}=0 \quad r=n+1, . ., N,
$$


with initial conditions $x_{r}(t)=1$. Hence, these new variables are constant and equal to 1 . Arbitrary powers of these $(\mathrm{N}-\mathrm{n})$ variables, $X_{r}(t), r=n+1, . ., N$ still equal to 1 , can be multiplied with the monomials $\prod_{k=1}^{n} X_{k}^{B_{j k}}$ that appear in (8). The previous monomials can, thus, be written $\prod_{k=1}^{N} X_{k}^{B_{j k}}$. In other word, the original Nxn rectangular matrix $\mathrm{B}$ has been completed to a square NxN matrix $\hat{B}$. If the rectangular matrix B is of maximal rank, then the completed square matrix $\hat{B}$ is always invertible. In the sequel we assume that the rank of $B$ is always maximal. Indeed, for the cases where the rank of $\mathrm{B}$ is not maximal, we refer to the article [5] where it is shown that the system can be decoupled into a lower dimensional QP system with a new matrix $\tilde{B}$ with maximal rank and another lower dimensional sub-system that is linear.

The whole system (8) has, thus, been transformed into a N-dimensional QP system with $n=N$ :

$$
\dot{x}_{i}=\hat{\alpha}_{i} x_{i} \sum_{j=1}^{N} \hat{A}_{i j} \prod_{k=1}^{n} x_{k}^{\hat{B}_{j k}} \quad i=1, \ldots, N,
$$

in which, to take into account equations (17), $\hat{\alpha}$ is the old vector $\alpha$ completed by $N-n$ lines of zero and $\hat{A}$ is the old rectangular matrix $A$ completed, also, by $N-n$ lines of zeroes.

Now, since system (18) has a number of monomials that is equal to the number of dimensions of the system, we can proceed as in the previous case $n=N$. The matrix $B^{-1}$ exists and we use it in order to transform the system (18) with the help of a quasi-monomial transformation of the form:

$$
\dot{x}_{i}=\prod_{k=1}^{N} X_{k}^{{ }^{\prime} \hat{B}_{j k}^{-1}} \quad i=1, \ldots, N,
$$

leading to:

$$
\dot{x}_{i}^{\prime}=\hat{\lambda} x_{i}^{\prime}+x_{i}^{\prime} \sum_{j=1}^{N} \hat{M}_{i j} x_{j}^{\prime} \quad i=1, \ldots, N,
$$

where

$$
\hat{M}=\hat{B} \circ \hat{A} \quad \text { and } \quad \hat{\lambda}=\hat{B} \circ \hat{\alpha} .
$$

However, by construction of the two new square matrices $\hat{A}$ and $\hat{B}$, and of the vector $\hat{\alpha}$, one has: $\hat{M}=\hat{B} \circ \hat{A}=$ $B \circ A=M$ and $\hat{\lambda}=\hat{B} \circ \hat{\alpha}=B \circ \alpha=\lambda$. We thus get:

$$
\dot{x}_{i}^{\prime}=\lambda x_{i}^{\prime}+x_{i}^{\prime} \sum_{j=1}^{N} M_{i j} x_{j}^{\prime} \quad i=1, \ldots, N .
$$

This is the same Lotka-Volterra system as in (16) but, now, in $\mathrm{N}$ dimensions. We have embedded the dynamics of our initially n-dimensional QP system into a Ndimensional LV system.
For the case $n>N$, we refer to the article [5] for the demonstration of the reduction to LV systems. In this case, the LV system found is again the same as in (22). But now, the embedding is replaced by a projection from the original n-dimensional phase-space into a LV system living in a smaller dimensional $(\mathrm{N})$ phase-space.

In fact, in all the three cases, the dimension of the LV system is the number $\mathrm{N}$ of independent monomials. Moreover, obviously, the new variables $x_{i}^{\prime}$ in terms of which the LV system is written are these monomial themselves. This is clear by inverting relation (19). Thus a simple trick to find immediately the LV system corresponding to a given QP system is to make the list of the independent monomials appearing in it (when the pre-factor $x_{i}$ has been first extracted) and to calculate the time derivative of these monomials using the original QP system. This leads, directly to the LV system (22) with $M$ and $\lambda$ respectively given by $M=B \circ A$ and $\lambda=B \circ \alpha$.

We can conclude by the following main theorem :

All QP systems $\dot{x}_{i}=\alpha_{i} x_{i} \sum_{j=1}^{N} A_{i j} \prod_{k=1}^{n} x_{k}^{B_{j k}}$, $i=1, \ldots, n$, whose solutions are limited to the positive orthant, can be reduced to the LV form $\dot{x}_{i}^{\prime}=\lambda_{i} x_{i}^{\prime}+x_{i}^{\prime} \sum_{j=1}^{N} M_{i j} x_{j}^{\prime}, i=1, \ldots, N$, with $M=B \circ A$ and $\lambda=B \circ \alpha$.

Furthermore, the infinite set of all the QP systems is divided into equivalence classes. Any such class contains a canonical simplest form, the LV system. Each class is characterized by the matrix $\mathrm{M}$ and the vector $\lambda=B \circ \alpha$ in the LV system of the class.

A last remark is that the type of nonlinearities occurring in a QP system is unessential since the LV system to which it is equivalent has always a quadratic nonlinearity.

\section{G. Extension of the set of dynamical systems that are reducible to QP systems}

The generality of the above theorem is further increased by the fact that nonlinear dynamical systems

$$
\dot{x}_{i}=f_{i}\left(x_{1}(t), \ldots, x_{n}(t)\right) \quad i=1, . ., n,
$$

that contain some nonlinear functions $f_{i}\left(x_{1}(t), \ldots, x_{n}(t)\right)$ which are more general than polynomial or quasipolynomial, can be re-casted in the form of QP systems [6, 7]. This is generally possible if the functions $f_{i}\left(x_{1}(t), \ldots, x_{n}(t)\right)$ are enough regular and are themselves solutions of polynomial or quasi-polynomial differential equations. Then these last equations can be added to the original system (23) to form a higher dimensional system that will be a QP system. By the main theorem shown above this new QP system can, in turn, be transformed into a LV system. The LV quadratic nonlinearity is the ultimate reduction of nonlinearity that can be reached by exact transformations. This fact considerably enlarges the field of the quasi-polynomial approach. 


\section{H. Applications: Invariants, dimensional reduction, stability}

\section{Exact integrability and reduction properties of QP and LV systems}

In some cases a QP system can be completely integrated or reduced to a smaller dimension. This happens when enough invariants can be found or when some transformations can decouple the system into several sub-systems, some of them being linear. This happens when, for instance, the matrix $\mathrm{A}$ or $\mathrm{B}$ are not of maximal rank. If matrix $\mathrm{A}$ has a rank, say $\mathrm{r}$, that is smaller than $\mathrm{n}$ (the maximal rank) then there exists a particular quasi-monomial transformation (11) that leads to find $n-r$ invariants. If, on the contrary it is $\mathrm{B}$ that is of non-maximal rank, then, a quasi-monomial transformation exists that decouples the transformed system into one independent nonlinear sub-system of smaller dimension and one linear sub-system that depends on the solutions of the former. Details of these properties and others that cannot be reported here are to extensive to be reported here. They can be found in the following publications [1, 8,10. Moreover, most of these algorithms are implemented in the computer algebra software [QPSI] written in the language MAPLE [11.

\section{Stability properties of QP and LV sys- tems}

For a LV system it is always possible to find some Lyapunov function that allows for studying the stability properties of its trajectories 2, 3]. Since we have shown that most nonlinear dynamical systems can be transformed into LV form, we can transfer all the stability properties of the latter to these more general dynamical systems. This has led to a large corpus of results, too large to be exposed here, that can be found in the following articles [12 17 .

\section{LECTURE 2}

In this lecture we now focus on the Lotka-Volterra systems in order to find some of their Lie algebraic properties and, finally, to find two forms of their general solutions. These results can, in turn, be transferred to the original quasi-polynomial systems by performing the appropriate inverse quasi-monomial transformations.

First, let us transform the LV system:

$$
\dot{x}_{i}=\lambda_{i} x_{i}+x_{i} \sum_{j=1}^{N} M_{i j} x_{j} \quad i=1, \ldots, N,
$$

into the $N+1$ dimensional system

$$
\dot{x}_{i}=x_{i} \sum_{j=1}^{N+1} \Omega_{i j} x_{j} \quad i=1, \ldots, N+1,
$$

where the matrix $\Omega$ is the following extension of matrix $\mathrm{m}$ :

$$
A=\left(\begin{array}{cc}
M_{11} \ldots M_{1 N} & \lambda_{1} \\
\ldots \ldots \ldots \ldots \ldots & \\
M_{N 1} \ldots M_{N N} & \lambda_{N} \\
\ldots \ldots \ldots \ldots \ldots \ldots &
\end{array}\right)
$$

with $X_{N+1}(t=0)=1$ so that $X_{N+1}(t)=1$ for all $t>0$.

\section{A. Carleman infinite embedding representation of LV dynamics}

The Carleman embedding [18 consists in embedding the original systems of ODEs into an infinite dimensional space in the following manner.

Define for the $N+1$ variables of (25) the following monomials:

$$
\psi\left(r_{1}, r_{2}, \ldots, r_{N+1} ; t\right)=X_{1}^{r_{1}}(t) X_{2}^{r_{2}}(t) \ldots X_{N+1}^{r_{N+1}}(t),
$$

with $r_{i}=0,1,2, \ldots$ for all $i=1, \ldots, N+1$.

Then, take the time derivative of (26) and use equation (25). This gives [19]:

$$
\partial_{t} \psi\left(r_{1}, r_{2}, \ldots, r_{N+1} ; t\right)=H \psi\left(r_{1}, r_{2}, \ldots, r_{N+1} ; t\right),
$$

with the pseudo-Hamiltonian:

$$
H \equiv \sum_{i=1}^{N+1} \sum_{j=1}^{N+1} r_{i} \Omega_{i j} D_{j},
$$

with the displacement operators $D_{j}$ given by:

$$
D_{j} f\left(r_{1}, r_{2}, \ldots, r_{N+1} ; t\right)=f\left(r_{1}, r_{2}, \ldots, r_{N+1} ; t\right) .
$$

Equation (28) looks like a Schrödinger equation, however, the pseudo-Hamiltonian operator is not Hermitian and, consequently, equation (28) cannot be associated to a true quantum system.

To be completely equivalent to the original LV problem (25), the equation (28) must be completed with the following particular initial conditions:

$$
\psi\left(r_{1}, r_{2}, \ldots, r_{N+1} ; 0\right)=X_{1}^{r_{1}}(0) X_{2}^{r_{2}}(0) \ldots X_{N+1}^{r_{N+1}}(0),
$$

where the $X_{i}(0)$ are the initial values of the $\mathrm{LV}$ equation (25).

Let us look more algebraically at the above result. Using definition (30), let us compute the commutators $\left[D_{i}, r_{j}\right]$ :

$$
\left[D_{i}, r_{j}\right]=D_{i} \delta_{i j} .
$$

Now let us go to the Heisenberg picture of the above pseudo-quantum problem (28-29) and calculate the time derivative of the operators $D_{i}(t)$ with the help of the pseudo-Hamiltonian H (29):

$$
\dot{D}_{i}(t)=\left[D_{i}(t), H\right] .
$$


This gives:

$$
\dot{D}_{i}(t)=D_{i}(t) \sum_{j=1}^{N+1} \Omega_{i j} D_{j}(t) \quad i=1, \ldots, N+1 .
$$

Equation (34) is just the Lotka-Volterra equation (25), but written here for the operators .

This leads us to the conclusion that the LV equation (25) (or (34)) and its Carleman embedding (28) are, respectively, the Heisenberg and the Schrödinger versions of the same pseudo-quantum system. The time evolution of this system is characterized by the pseudo-Hamiltonian $\mathrm{H}$ given in (29).

\section{B. Abstract Lie algebraic structure of LV systems}

Now, let us generalize the above notions. We define two sets of abstract operators:

$$
\left(A_{i} ; i=1, \ldots, N+1\right) \quad \text { and } \quad\left(B_{i} ; i=1, \ldots, N+1\right),
$$

with the following commutation rules:

$$
\left[A_{i}, A_{j}\right]=0, \quad\left[B_{i}, B_{j}\right]=0, \quad\left[B_{i}, A_{j}\right]=B_{i} \delta_{i j} .
$$

With these operators let us define a pseudo-Hamiltonian operator:

$$
H \equiv \sum_{i=1}^{N+1} \sum_{j=1}^{N+1} A_{i} \Omega_{i j} B_{j},
$$

where the constant coefficients $\Omega_{i j}$ are the coupling constants of the Lotka-Volterra system (25).

Then, we have the time evolution in the Heisenberg picture:

$\dot{B}_{i}(t)=\left[B_{i}(t), H\right]=B_{i} \sum_{j=1}^{N+1} \Omega_{i j} B_{j} \quad i=1, \ldots, N+1$,

and the time evolution of the same system in the Schrödinger picture:

$$
\partial_{t} \psi(t)=H \psi(t) .
$$

The time evolution of the operators $A_{i}$ can be easily calculated and obeys a system of non-autonomous linear differential equations of no interest here.

The equation (37) is just the abstract form of the $\mathrm{LV}$ system for the operators $B_{i}$, while the equation (38) is the abstract form of the pseudo-Schrödinger equation (28). These equations are independent of the explicit realization of the operators $A_{i}$ and $B_{i}$. In fact, the operators $D_{i}$ and $r_{i}(i=1, \ldots, N+1)$ were one instance of such a realization with $A_{i}=r_{i}, B_{i}=D_{i}$. We shall now present two others realizations of the abstract operators $A_{i}$ and $B_{i}$.

\section{Two other realizations of the $\mathbf{L V}$ abstract Lie algebra: creation-destruction operators and Liouville equation}

\section{Creation destruction operator realization}

Let us now realize the above operators $\mathrm{Ai}$ and $\mathrm{Bi}$ in terms of boson creation-destruction operators $a_{i}, a_{i}^{+}$. The latter obey the usual boson commutation rules:

$\left[a_{i}, a_{j}^{+}\right]=\delta_{i j}, \quad\left[a_{i}, a_{j}\right]=0, \quad\left[a_{i}^{+}, a_{j}^{+}\right]=0 \quad i=1, \ldots, N+1$.

We now identify the operators $A_{i}$ with the number operators $a_{i}^{+} a_{i}$ and the operators $B_{i}$ with the destruction operators :

$$
A_{i}=a_{i}^{+} a_{i}, \quad B_{i}=a_{i} .
$$

With the help of the commutators (39), it is easy to check that the commutation rules (35) are verified for the $A_{i}$ 's and the $B_{i}$ 's.

The abstract pseudo-Hamiltonian (36) now becomes:

$$
H \equiv \sum_{i=1}^{N+1} \sum_{j=1}^{N+1} a_{i}^{+} a_{i} \Omega_{i j} a_{j}
$$

Let us calculate the time derivative of the ai $(\mathrm{t})$ operators in the Heisenberg picture:

$$
\dot{a}_{i}(t)=\left[a_{i}(t), H\right] .
$$

This is readily done and gives:

$$
\dot{a}_{i}(t)=a_{i}(t) \sum_{j=1}^{N+1} \Omega_{i j} a_{j}(t) .
$$

This is exactly the LV system for the time evolution of the destruction operators $a_{i}, i=1, \ldots, N+1$. The time evolution of the operators $A_{i}=a_{i}^{+} a_{i}$ is governed by linear differential equations that are not relevant here.

One could also calculate the time evolution in the Schrödinger picture but is not interesting for our next purposes.

Though very similar to quantum physical systems, the system characterized by the above pseudo-Hamiltonian (41) is not physical: It is not hermitian and does not preserve the number of "particles "in time. But, of course, we do not expect a general nonlinear dynamical system to be equivalent to a quantum mechanical system!

\section{Liouvillian realization}

Let us now consider the following realization of the abstract operators $A_{i}$ and $B_{i}(i=1, \ldots, N+1)$ :

$$
A_{i} \equiv-\frac{\partial}{\partial X_{i}} X_{i}, \quad B_{i} \equiv X_{i} .
$$

The commutation rules (35) can easily be verified using the elementary rules of derivative calculation. 
The pseudo-Hamiltonian (36) becomes here:

$$
H \equiv-\sum_{i=1}^{N+1} \sum_{j=1}^{N+1} \frac{\partial}{\partial X_{i}} X_{i} \Omega_{i j} X_{j}
$$

In the Heisenberg picture, the time evolution of the operators $x_{i}(t)$ :

$$
\dot{x}_{i}(t)=\left[x_{i}(t), H\right] \quad i=1, \ldots, N+1,
$$

is easily shown to give :

$$
\dot{x}_{i}(t)=x_{i} \sum_{j=1}^{N+1} \Omega_{i j} x_{j} \quad i=1, \ldots, N+1,
$$

which is exactly the Lotka-Volterra system.

In the Schrödinger picture, using equation (38), we get

$$
\partial_{t} \psi\left(r_{1}, r_{2}, \ldots, r_{N+1} ; t\right)=-\sum_{i=1}^{N+1} \sum_{j=1}^{N+1} \frac{\partial}{\partial X_{i}} X_{i} \Omega_{i j} X_{j} \psi\left(r_{1}, r_{2}, \ldots, r_{N+1} ; t\right)
$$

This partial derivative equation is easily recognizable. It is the Liouville equation associated to the LV system. It governs the time evolution of the probability density $\psi\left(r_{1}, r_{2}, \ldots, r_{N+1} ; t\right)$ of the point representing the position at time t of the LV system in the $N+1$ phase-space. The probability density at time zero must be a given distribution function $\psi_{0}$ :

$$
\psi\left(r_{1}, r_{2}, \ldots, r_{N+1} ; 0\right)=\psi_{0}\left(r_{1}, r_{2}, \ldots, r_{N+1}\right) .
$$

Below, we shall use this realization in order to derive a path integral form of the general solution of a LV system.

\section{The abstract Lie algebraic structure of linear and LV dynamical systems}

Lets us assume we have two dynamical systems, one linear and one $\mathrm{LV}$, in $\mathrm{N}+1$ dimensions phase-space. We can describe both types of systems with sets of operators $A_{i}, B_{i}$.

For linear systems, these operators must satisfy the following commutation rules :

$$
\left[A_{i}, A_{j}\right]=0, \quad\left[B_{i}, B_{j}\right]=0, \quad\left[B_{i}, A_{j}\right]=\delta_{i j} .
$$

For LV systems, these operators must satisfy the commution rules already given in (35):

$$
\left[A_{i}, A_{j}\right]=0, \quad\left[B_{i}, B_{j}\right]=0, \quad\left[B_{i}, A_{j}\right]=B_{i} \delta_{i j} .
$$

For both type of systems the pseudo-Hamiltonian has the same expression in terms of these operators:

$$
H \equiv \sum_{i=1}^{N+1} \sum_{j=1}^{N+1} A_{i} \Omega_{i j} B_{j}
$$

It can easily be checked, using respectively commutation rules (50) and (51), that the Heisenberg time evolution equations for the $N+1$ operators $\mathrm{Bi}$ :

$$
\dot{B}_{i}(t)=\left[B_{i}(t), H\right],
$$

yields:

Linear system :

$$
\dot{B}_{i}(t)=\sum_{j=1}^{N+1} \Omega_{i j} B_{j}
$$

LV system :

$$
\dot{B}_{i}(t)=B_{i} \sum_{j=1}^{N+1} \Omega_{i j} B_{j}
$$

Thus, the only difference in the Lie algebra of both types of systems resides in the third commutator in both expressions (50) and (51). The evaluate the generality of this result, one should keep in mind that the LV system represents, in fact, a whole class of equivalence of nonlinear dynamical systems as we have shown earlier in this presentation.

\section{E. General solution via Taylor series}

In order to derive the Taylor series solution of the LV system (25), the most appropriate realization is that generated by the operators $r_{i}$ and $D_{i}$ in equations (27-34). Indeed, let us first combine the equations (28) and (29). This gives:

$$
\partial_{t} \psi\left(r_{1}, r_{2}, \ldots, r_{N+1} ; t\right)=\sum_{i=1}^{N+1} \sum_{j=1}^{N+1} r_{i} \Omega_{i j} D_{j} \psi\left(r_{1}, r_{2}, \ldots, r_{N+1} ; t\right)
$$


with the particular initial condition:

$$
\psi\left(r_{1}, r_{2}, \ldots, r_{N+1} ; 0\right)=X_{1}^{r_{1}}(0) X_{2}^{r_{2}}(0) \ldots X_{N+1}^{r_{N+1}}(0) .
$$

The Taylor series solution of (56) is expressed by:

$$
\psi(\bar{r}, t)=\sum_{k=0}^{\infty} \frac{t^{k}}{k !} c_{k}(\bar{r}),
$$

where $\bar{r}$ denotes the vector $\left(r_{1}, \ldots, r_{N+1}\right)$ and the coefficients of the series, $c_{k}(\bar{r})$, are given by:

$$
c_{k}(\bar{r})=\left.\frac{d^{k}}{d t^{k}} \psi(\bar{r}, t)\right|_{t=0},
$$

which, with the help of equation (56) iterated $\mathrm{k}$ time, leads to:

$$
c_{k}(\bar{r})=\left(\sum_{i=1}^{N+1} \sum_{j=1}^{N+1} r_{i} \Omega_{i j} D_{j}\right)^{k} \psi(\bar{r} ; 0)
$$

that is, the k-th power of the Hamiltonian acting on the initial $\psi$ function.

Using the definition (30) of the displacement operators $D_{i}$ and the expression (57) of the initial function, one gets without difficulty:

$$
c_{k}(\bar{r})=\left[\prod_{j=1}^{N+1} X_{j}^{r_{j}}(0)\right] \sum_{i_{1}=1}^{N+1} \ldots \sum_{i_{k}=1}^{N+1}(r \Omega)_{i_{1}}\left((r \Omega)_{i_{2}}+(\Omega)_{i_{1} i_{2}}\right) \ldots\left(\left((r \Omega)_{i_{k}}+(\Omega)_{i_{1} i_{k}}+\ldots+(\Omega)_{i_{k-1} i_{k}}\right) X_{i_{1}}(0) \ldots X_{i_{k}}(0) .\right.
$$

To obtain the Taylor series solution of the LV system itself, one must remember that

$$
X_{i}=\psi\left(\bar{r}=e^{i}, t\right),
$$

where

$$
e^{i}=\left(0, \ldots, 0, r_{i}=1,0, \ldots 0\right) .
$$

Thus, the Taylor series solution of the LV system:

$$
x_{i}=\sum_{j=1}^{N+1} \Omega_{i j} x_{j},
$$

is given by :

$$
X_{i}(t)=\sum_{k=0}^{\infty} \frac{t^{k}}{k !} c_{k}\left(e^{i}\right),
$$

with the following expression of the general coefficient :

$$
c_{k}\left(e^{i}\right)=X_{j}(0) \sum_{i_{1}=1}^{N+1} \ldots \sum_{i_{k}=1}^{N+1}(r \Omega)_{i_{1}}\left((r \Omega)_{j_{2}}+(\Omega)_{i_{1} i_{2}}\right) \ldots\left(\left((r \Omega)_{i_{k}}+(\Omega)_{i_{1} i_{k}}+\ldots+(\Omega)_{i_{k-1} i_{k}}\right) X_{i_{1}}(0) \ldots X_{i_{k}}(0) .\right.
$$

The solution $(65,66)$ of the LV system (64) defines a new class of special functions [19. This is in complete analogy with the generalized hypergeometric functions 20] that are defined as Taylor series giving the solutions of second order linear differential equations.

The fact, that we have obtained an analytic expression of the general term of the Taylor series gives much more information on the solution than the usual numerical Taylor expansions that are currently used to solve nonlinear differential systems. First, it allows for developing a computer algebra software for solving non-numerically
LV systems 21]. The program computes and stores results that are dependent of the symbolic values of the coefficients. This permits to vary the parameters at will without re-beginning the whole calculations.

Also, the knowledge of the general term of a Taylor series permits to evaluate the radius of convergence, to calculate analytical continuations and, also, to derive asymptotic expansions for the solution. However, this last claim is only in its infancy and must be developped.

One should, however, recognize that applications of this form of the solution are up to now limited by the 
difficulty of finding closed expressions of the many finite sums appearing in the expression (66) of the Taylor coefficients. The complexity of these sums reflects all the complexity of the nonlinear dynamical systems! It is comparable to the complexity of the partition function of a system of $\mathrm{N}$ particles interacting through a binary interaction potential, at thermal equilibrium. The partition function is also expressed in terms of many finite sums of a summand that depends on the binary interaction potential between the $\mathrm{N}$ particles and its exact closed expression is usually out of our present technical ability.
However, expression (66) provides an alternative to other methods that could help obtaining more informations on the behaviour of the solutions of nonlinear dynamical systems.

\section{F. General solution via path integrals}

The Liouvillian realization of the abstract LV algebra is better adapted for the objective of deriving a path integral form of the solution of the LV system (25).

We recall the description of the time evolution of the system in Schrödinger picture:

$$
\partial_{t} \psi\left(X_{1}, X_{2}, \ldots, X_{N+1} ; t\right)=-\sum_{i=1}^{N+1} \sum_{j=1}^{N+1} \frac{\partial}{\partial X_{i}} X_{i} \Omega_{i j} X_{j} \psi\left(X_{1}, X_{2}, \ldots, X_{N+1} ; t\right)
$$

For the Liouville equation (67) to be completely equivalent to the original deterministic LV equation (25), we need to assume that the initial distribution is deterministic, i.e., the initial condition is known with probability one :

$$
\psi\left(X_{1}, X_{2}, \ldots, X_{N+1} ; 0\right)=\prod_{i=1}^{N+1} \delta\left(X_{i}-X_{i}^{0}\right)
$$

$$
\psi\left(X_{1}, X_{2}, \ldots, X_{N+1} ; t\right)=<\prod_{i=1}^{N+1} \delta\left(X_{i}-X_{i}\left(t, X_{1}(0), \ldots, X_{N+1}(0)\right)\right)>_{\psi(t=0)}
$$

where the symbol \langle\rangle$_{\psi(t=0)}$ means the operation of average over the initial distribution $\psi(\bar{X} ; t=0)$.
The formal solution of equation (67), for any initial distribution, can always be written as :

$\psi\left(X_{1}, X_{2}, \ldots, X_{N+1} ; t\right)=\int d X_{1}(0) \ldots d X_{N+1} \prod_{i=1}^{N+1} \delta\left(X_{i}-X_{i}\left(t, X_{1}(0), \ldots, X_{N+1}(0)\right)\right) \psi\left(X_{1}(0), X_{2}(0), \ldots, X_{N+1}(0) ; t=0\right)$

The explicit expression of the average in (69) gives:

where $X_{i}\left(t, X_{1}(0), \ldots, X_{N+1}(0)\right),(i=1, \ldots, N+1)$, denotes the trajectory of the solution to (25).
Let us now consider the expression (70) for a short time $\Delta t \equiv t / n$ :

$\psi\left(X_{1}, X_{2}, \ldots, X_{N+1} ; \Delta t\right)=\int d X_{1}(0) \ldots d X_{N+1} \prod_{i=1}^{N+1} \delta\left(X_{i}-X_{i}\left(\Delta t, X_{1}(0), \ldots, X_{N+1}(0)\right)\right) \psi\left(X_{1}(0), X_{2}(0), \ldots, X_{N+1}(0) ; t=0\right)$

For large enough values of $\mathrm{n}$, that is, for small enough values of, the solution of the LV system (25) can be 
written:

$X_{i}\left(\Delta t, X_{1}(0), \ldots, X_{N+1}(0)\right)=X_{i}(0)+\Delta t X_{i}(0) \sum_{j=1}^{N+1} \Omega_{i j} X_{i}(0)$
Inserting equation (72) into (71), we get:

$$
\begin{aligned}
& \psi\left(X_{1}, X_{2}, \ldots, X_{N+1} ; \Delta t\right)=\int d X_{1}(0) \ldots d X_{N+1}(0) \times \\
& \prod_{i=1}^{N+1} \delta\left(X_{i}-X_{i}(0)-\Delta t X_{i}(0) \times\right. \\
&\left.\sum_{j=1}^{N+1} \Omega_{i j} X_{i}(0)\right) \psi\left(X_{1}(0), X_{2}(0), \ldots, X_{N+1}(0) ; t=0\right) .
\end{aligned}
$$

$i=1, \ldots, N+1$.

If we displace the initial time from $t=0$ to $t=\Delta t$ in (73), we obtain:

$\psi\left(X_{1}, X_{2}, \ldots, X_{N+1} ; 2 \Delta t\right)=\int d X_{1}(1) \ldots d X_{N+1}(1) \prod_{i=1}^{N+1} \delta\left(X_{i}-X_{i}(1)-\Delta t X_{i}(1) \sum_{j=1}^{N+1} \Omega_{i j} X_{i}(1)\right) \psi\left(X_{1}(1), X_{2}(1), \ldots, X_{N+1}(1) ; \Delta t\right)$

where we used the notation:

$$
X_{i}(k) \equiv X_{i}(k \Delta t)
$$

The relation (74) can be translated up to time $t=n \Delta t$ :

$$
\begin{aligned}
& \psi\left(X_{1}, X_{2}, \ldots, X_{N+1} ; n \Delta t\right)=\int d X_{1}(n-1) \ldots d X_{N+1}(n-1) \times \\
& \prod_{i=1}^{N+1} \delta\left(X_{i}-X_{i}(n-1)-\Delta t X_{i}(n-1) \sum_{j=1}^{N+1} \Omega_{i j} X_{i}(n-1)\right) \times \\
& \psi\left(X_{1}(n-1), X_{2}(n-1), \ldots, X_{N+1}(n-1) ;(n-1) \Delta t\right) .(76)
\end{aligned}
$$

The recursion on $\mathrm{n}$ appearing in (75) can now be iterated down to $n=0$, leading to:

$$
\begin{aligned}
& \psi\left(X_{1}, X_{2}, \ldots, X_{N+1} ; n \Delta t\right)=\int d^{N+1} X_{1}(n-1) \ldots d^{N+1} X_{N+1}(0) \times \\
& \prod_{i_{n-1}=1}^{N+1} \delta\left(X_{i_{n-1}}-X_{i_{n-1}}(n-1)-\Delta t X_{i_{n-1}}(n-1) \sum_{j_{n-1}=1}^{N+1} \Omega_{i_{n-1} j_{n-1}} X_{j_{n-1}}(n-1)\right) \times \\
& \prod_{i_{n-2}=1}^{N+1} \delta\left(X_{i_{n-2}}(n-2)-X_{i_{n-2}}(n-2)-\Delta t X_{i_{n-2}}(n-2) \sum_{j_{n-2}=1}^{N+1} \Omega_{i_{n-2} j_{n-2}} X_{j_{n-2}}(n-2)\right) \ldots \\
& \prod_{i_{0}=1}^{N+1} \delta\left(X_{i_{0}}(1)-X_{i_{0}}(0)-\Delta t X_{i_{0}}(0) \sum_{j_{0}=1}^{N+1} \Omega_{i_{0} j_{0}} X_{j_{0}}(0)\right) \psi\left(X_{1}(0), X_{2}(0), \ldots, X_{N+1}(0) ; 0\right) .
\end{aligned}
$$

Obviously, the $\mathrm{n}$ integrals appearing in (76) represent a summation over all the possible (broken) paths connecting the point $\bar{X}(0)$ to the point $\bar{X}(n \Delta=t) \equiv \bar{X}$ in the $\mathrm{N}+1$-dimensional phase-space of the LV system (25). In the limit $n \rightarrow \infty, \Delta t=0, n \Delta=t$ (t finite), these paths become smooth curves and the $\mathrm{n}$ integrals tend towards a so-called path integral.

As a last step, we introduce the particular initial con- dition (68) in the expression (76). This enables us to perform the integral over all the possible positions of the initial point $\bar{X}(0)$. This operation along with the following property of the Dirac delta distribution:

$$
\delta(a x)=\frac{1}{|a|} \delta(x)
$$

transforms the expression (76) into: 


$$
\begin{gathered}
\psi\left(X_{1}, X_{2}, \ldots, X_{N+1} ; n \Delta t\right)=\frac{1}{\Delta t^{n(N+1)}} \int d^{N+1} X_{1}(n-1) \ldots d^{N+1} X_{N+1}(0) \times \\
\prod_{i_{n-1}=1}^{N+1} \delta\left(\frac{X_{i_{n-1}}-X_{i_{n-1}}(n-1)}{\Delta t}-X_{i_{n-1}}(n-1) \sum_{j_{n-1}=1}^{N+1} \Omega_{i_{n-1} j_{n-1}} X_{j_{n-1}}(n-1)\right) \times \\
\prod_{i_{n-2}=1}^{N+1} \delta\left(\frac{X_{i_{n-2}}(n-2)-X_{i_{n-2}}(n-2)}{\Delta t}-X_{i_{n-2}}(n-2) \sum_{j_{n-2}=1}^{N+1} \Omega_{i_{n-2} j_{n-2}} X_{j_{n-2}}(n-2)\right) \ldots \\
\prod_{i_{0}=1}^{N+1} \delta\left(\frac{X_{i_{0}}(1)-X_{i_{0}}^{0}}{\Delta t}-X_{i_{0}}^{0} \sum_{j_{0}=1}^{N+1} \Omega_{i_{0} j_{0}} X_{j_{0}}^{0}\right) .
\end{gathered}
$$

One observes in (78) the apparition of the component $\mathrm{i}$ of the discretized velocity at time $k \Delta t:\left(X_{i}(k)-X_{i}(k-\right.$ 1)) $/ \Delta t$. Taking the limit $n \rightarrow \infty, \Delta t=0, n \Delta=t(\mathrm{t}$ finite) of (78), we obtain the path integral form of the solution to the LV system (25):

$\psi(\bar{x}, t)=\int_{\bar{x}(0)}^{\bar{x}=\bar{x}(t)} D x(\cdot) \prod_{i=1}^{N+1} \delta\left(\dot{x}_{i}(\cdot)-x_{i}(\cdot) \sum_{j=1}^{N+1} \Omega_{i j} x(\cdot)\right)$

where the path integral operator is defined as:

$$
\lim _{n \rightarrow \infty, \Delta t=0, n \Delta=t} \frac{1}{\Delta t^{n(N+1)}} \int d^{N+1} X_{1}(n-1) \ldots d^{N+1} X_{N+1}(0)
$$

and where $x_{i}(\cdot)$ represents the function $x_{i}$ of time as a point in a set of functions.

The path integral (79) is a new result. It is a very complex mathematical object. Usually, in order to compute such path integrals, one needs to go back to the discrete time version. Here, it means to return to equation (78). After completing the calculations, one takes the limit $n \rightarrow \infty, \Delta t=0, n \Delta=t$. There is no guaranty up to now that such calculations are feasible but I think them worth to be attempted.

Of course all the results exposed in the two last chapters can be back transformed to the original QP systems. We do not present this inverse transformation here because it is quite simple.

\section{CONCLUSION}

I hope to have convinced the reader that the QP theory is a significant progress in the study of nonlinear dynamical systems. It unifies the all domain around the study of the unique Lotka-Volterra canonical form. This offers the possibility to transfer all the mathematical knowledge about LV systems to the much larger domain of the nonlinear dynamical systems that can be put in the quasi-polynomial format. This transfer is far from being completed by now but it would be of high interest to achieve it because the amount of results known on the LV systems is really huge.

Furthermore, I have shown that the LV format leads to a deeper understanding of the Lie algebraic structure that lies underneath the linear and LV systems, and more generally nonlinear dynamical systems. This new, more algebraic, way of looking at dynamical systems seems promising. It provides a new flexibility by allowing for , choosing among various realizations of the same dynamical system, the one that is more adapted to one's objective.

The algebraic point of view permits also to discover a deep similarity between dynamical systems that are, after all, classical systems and quantum systems. Among the quantum tools that can be applied to classical dynamical systems we have the different pictures of the time evolution of a dynamical system such as the Heisenberg and Schrödinger pictures. We have found also that a dynamical system is always generated by a Hamiltonian operator. When written in terms of creation-destruction boson operators, the similarity of these Hamiltonians with those of quantum systems becomes striking.

Among the various realizations of the algebra of $\mathrm{LV}$ systems we showed that two of them, at least, are enough practical to obtain a form of the general solution. These solutions are quite complex. This is expected for nonlinear dynamical systems that can, for instance, be in a chaotic regime.

A deep question remains: What happens to these solutions when a given dynamical system is in such a chaotic regime? The answer to this question needs a way to extract from one or another form of the general solution its time asymptotic behaviour. This is a very exciting work that still remains to be done!

[1] L.Brenig: Complete factorisation and analytic solutions of generalized Lotka-Volterra equations, Phys.Lett.

A133, 378 (1988). 
[2] Y.Takeuchi: Global Dynamical Properties of LotkaVolterra systems (World Scientific, 1994).

[3] S.Baigent, Lotka-Volterra Dynamics-An introduction, http://www.Itcc.ac.uk/courses/BioMathematics/ LTCC_LV2010.pdf (2010).

[4] B.Hernández-Bermejo, V.Fairén and L.Brenig: Algebraic recasting of nonlinear systems of ODEs into universal formats, J.Phys.A: Math.Gen. 31, 2415-2430 (1998).

[5] L.Brenig, A.Goriely: Universal canonical forms for timecontinuous dynamical systems, Phys.Rev.A 40, 4119 (1989).

[6] E.H.Kerner: Universal formats for nonlinear differential systems, J.Math.Phys. 22, 1366, (1981).

[7] B.Hernández-Bermejo and V.Fairén: Lotka-Volterra representation of general nonlinear systems, Math.Biosci. 140, 1-32, (1997).

[8] A.Goriely and L.Brenig: Algebraic degeneracy and partial integrability for systems of ordinary differential equations, Physics Letters A 145, 245 (1990).

[9] A.Figueiredo, T.M.Rocha Filho and L.Brenig: Algebraic structures and invariant manifolds of differential systems, J.Math.Phys. 39, 2929 (1998).

[10] A.Figueiredo, T.M.Rocha Filho and L.Brenig: Necessary conditions for the existence of quasi-polynomial invariants: the quasi-polynomial invariants and Lotka-Volterra systems., Physica A, 262, 158 (1999).

[11] T. M. Rocha Filho, A. Figueiredo, L. Brenig : [QPSI] a Maple package for determination of quasi-polynomial symmetries and invariants, Computer Physics Communications 117, 263 (1999)

[12] A. Figueiredo, I. M. Gléria, T. M. Rocha Filho :
Boundness of solutions and Lyapunov functions in quasipolynomial systems, Physics Letters B 268, 335 (2000).

[13] I. M. Gléria, A. Figueiredo, T. M. Rocha Filho : Stability properties of a general class of nonlinear dynamical systems, Journal of Physics A 34, 1 (2001).

[14] B. Hernández-Bermejo : Stability conditions and Liapunov functions for quasi-polynomial systems, Appl.Math.Letters 15, 25-28 (2002).

[15] I. M. Gléria, A. Figueiredo, T. M. Rocha : A numerical method for the stability analysis of quasi-polynomial vector fields, Nonlinear Analysis - Theory, Methods and Applications 52, 329 (2003).

[16] T. M. Rocha Filho, I. M. Gléria, A. Figueiredo : A novel approach for the stability problem in non-linear dynamical systems, Computer Physics Communications 155, 21 (2003).

[17] T. M. Rocha Filho, I. M. Gléria, A. Figueiredo, L. Brenig : The Lotka-Volterra canonical format, Ecological Modelling 183, 95 (2005).

[18] Brenig L. and Fairen V : Analytic approach to initialvalue problems in nonlinear systems, J. Math. Phys. 22, 649-652 (1981).

[19] L.Brenig : New special functions solving nonlinear autonomous dynamical systems, arXiv: 0910.2584 (2009).

[20] A.M.Mathai, R.K.Saxena: Generalized Hypergeometric Functions with Applications in Statistics and Physical Sciences, Lecture Notes in Mathematics vol. 348, Springer (1973).

[21] M. Codutti : NODES : A Nonlinear Ordinary Differential Equations Solver, Proceedings of ISSAC'92 (International Symposium on Symbolic and Algebraic Computation), (Berkeley, 1992). 